\title{
El Instituto Lingüístico de Verano y la Educación Bilingüe en el Perú:
}

\author{
Una perspectiva
}

\author{
Bárbara Trudell*
}

The involvement of the Instituto Linguirstico de Verano (Summer Institute of Linguis(ics, ILV) in bilingual education in the Peruvian jungle has since its inception been based on a policy of close collaboration with the Peruvian Ministry of Education. The following paper oxamines ILV's philosophy of bilingual education as it has developed in the last 43 years of flcld work among Peru's jungle ethnic groups. Topics discussed include the underlying preIuppositions that have shaped ILV's work in bilingual education, ILV's prespective on bicultural and intercultural education, and an assessment of the advantages and disadvantages of the model of bilingual education supported by ILV.

L'engagement de l' Instituto Lingüístico de Verano (Institut Linguistique d'été - ILV) dans l' éducation bilingue á la jungle péruvienne, a été dès son début basé sur une politique d'étroite collaboration avec le Ministère d" Education péruvien. L' article qui suir examine la philosophie d'éducation bilingúe de l' ILV, telle qu' elle a été développee pendant 43 de travail parmi les groupes ethniques de la jungle péruvienne. Les thèmes discutés comprennent les présuppositions sous-jacentes qui ont informé le travail d'éducation bilingue de l' ILV, le point de vue de l'ILV sur l'éducation biculturelle et intercurturelle, et une ćvaluation des avantages et désavantages du modèle $d^{\prime}$ éducation bilingue appuyé par I'ILV.

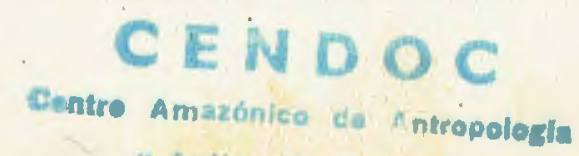


La participación del Instituto Lingüístico de Verano (ILV) en la educación bilingüe en la Amazonía Peruana ha tenido siempre el carácter de un esfuerzo mancomunado con el Ministerio de Educación. El primer convenio entre dicho ministerio y el ILV, firmado en 1945, comprometía a las dos entidades signatarias a "desarrollar un programa de cooperación para la investigación de las lenguas indigenas de la República" (Convenio Ministerio de Educación/Instituto Lingüístico de Verano, 1945). Desde ese entonces, el ILV ha continuado colaborando con diferentes gobiemos en lo que se refiere a la investigación lingüística, la implementación de programas educativos y la revaloración de las lenguas y las culturas indigenas.

En los primeros años, la infraestructura educativa era muy incipiente en las zonas donde se hablan los idiomas de los grupos minoritarios que habitan la Selva. Por esa razón, los trabajadores de campo del ILV se vieron precisados a involucrarse en la aplicación de las normas educativas del país entre esos grupos minoritarios. Con el correr de los años, la infraestructura oficial se amplió y los programas y la supervisión fueron perfeccionándose.

El examen cronológico de los convenios entre el ILV y el Ministerio de Educación nos da una visión de cómo se desarrolló la colaboración entre la entidad estatal y el ILV en lo que se refiere a la educación bilingüe en la Amazonía. La Resolución Suprema № 909 del 28 de noviembre de 1952, autorizó la creación de "cursos de capacitación para nativos alfabetizadores de la Selva Peruana" que debian llevarse a cabo en Yarinacocha (que entonces pertenecía al departamento de Loreto) y cuyo personal docente estaría conformado por educadores del ministerio y del ILV. En 1957, se creó el cargo de Coordinador del Plan Piloto de Educación Selvática y de las Escuelas Bilingủes y Rurales de la Selva, posición que fue cubierta por el Ministerio de Educación (Resolución Suprema No 1 del 3 de enero de 1957). En 1958, las escuelas bilingües de la Selva recibieron valor oficial y quedaron bajo la supervisión de la superintendencia de la Educación Primara en el Oriente (Decreto Supremo N ${ }^{2} 12$ del 28 de abril de 1958). En 1964, el Director de Educación Primaria y del Adulto aprobó el Reglamento de los Cursos de Capacitación para Maestros Bilingües de la Selva; y mediante la Resolución Ministerial $N^{2} 868$ del 28 de febrero de ese año, se autorizó al dinector del curso de capacitación a otorgar "el certificado oficial de Quinto Arto de Educación Primaria" a los maestros bilingües que hubieran estudiado seis niveles del curso. En 1969, se inició un programa de educación secundaria para los profesores que habian terminado primaria.

Con el inicio de la descentralización administrativa en el año 1972 como parte de la Reforma Educativa (Ley General de Educación, Decreto Ley № 19326 de marzo de 1972), las oficinas regionales empezaron a tener una mayor participación en la aplicación de las normas educativas. El rol del ILV pasóa ser más bien de asesoramiento. En los años siguientes se dieron varios pasos decisivos para el desarrollo de la educación bilingüe. Entre los más importantes mencionaremos los siguientes: la creación de la Unidad de Educación Bilingüe en el Ministerio de Educación, en 1973; la creación de un programa experimental de educación superior para los maestros bilingües, en 1983; y la oficialización de ese programa como el Instituto Superior Pedagógico Bilingüe, en 1985. 
El rol del ILV en el programa oficial de educación bilingüe de la Selva ha ido variando a lo largo de los años, como se puede notar al considerar los párrafos anteriores. Pero aunque las características específicas de esa paticipación han variado, dos compromisos han permanecido constantes: la colaboración estrecha con el organismo educativo oficial, y el afán de servir en lo posiblea los grupos nativos de la Amazonía.

En las páginas siguientes se hará un examen de la filosofía que ha servido de base a la participación del ILV en la educación bilingüe durante los cuarentitrés años de trabajo de campo entre los grupos étnicos de la Amazonía. Entre los tópicos que trataremos están las premisas que han determinado el carácter del trabajo del ILV en la educación bilingüe, la perspectiva del ILV en cuanto a la educación bilingüe intercultural, y una evaluación de los puntos positivos y negativos del modelo de educación bilingüe en el cual el ILV ha venido participando.

\section{Premisas fundamentales}

La premisa principal que sirve de base al trabajo del ILV en la educación bilingüe es el respeto a los grupos étnicos, pues considera que "todos los seres humanos nacen libres e iguales en dignidad y derechos"; tienen el derecho a la libertad, la seguridad personal la educación (Declaración Universal de Derechos Humanos, artículos 1, 4, 26). Es decir, que no se les debe privar del derecho de recibir educación por causa de su lengua y su cultura; al contrario, deben tener acceso a una educación que sea apropiada desde el punto de vista lingüístico y cultural. En su compromiso de colaboración con el Ministerio de educación, el ILV busca "el rescate, revalorización y desarrollo de las lenguas y culturas autóctonas, evitando su deterioro y desaparición" (Dirección General de Educación Bilingüe, 1989:11)

La importancia de la lengua vernácula para la preservación de la cultura nativa es otra de las premisas fundamentales del ILV:

El lenguaje es una de las partes sumamente importantes y positivas de una cultura, ya que constituye tanto un vehículo como una parte substancial de ésta. Es un rasgo clave para la preservación de la unidad e identidad dẹ los individuos de una cultura. Por lo tanto, el que su lengua sea considerada digna de ser empleada como medio educativo y el que su herencia cultural oral sea merecedora de ser preservada y difundida mediante la escritura son una contribución a sus expectativas y a su dignidad y respeto propio. Cuando los nuevos alfabetizados pueden expresarse por escrito, no sólo poseen medios para su autorrealización y autoidentificación sino que además tienen la satisfacción de contribuir a la preservación de sus valores culturales escribiendo sus historias y tradiciones. (Larson, Davis y Ballena Dávila, 1979: 415-6).

El ILV , asimismo, reconoce que el contacto intercultural entre los grupos autóctonos y la cultura mayoritaria es inevitable. En más de cuarenta aftos de trabajo entre las comunidades nativas de la Selva, el ILV ha sido testigo de los resultados del 
contacto de casi todos los grupos étnicos con la cultura mestiza -contacto que comenzó en la primera parte del presente siglo o aún más temprano, mucho antes de la llegada de los trabajadores de campo del ILV. Muchos de los contratos tempranos han sido desventajosos para las culturas indígenas, pues las realizaron conquistadores, colonizadores y personas interesadas en explotar las riquezas naturales que esclavizaron, explotaron, vendieron y masacraron a los grupos nativos que encontraron. Un lider aguaruna habla de los primeros contactos con foráneos:

Mientras la sociedad aguaruna vivía en esta forma llegaron los blancos en busca de caucho, oro, pieles finas y otros productos para su riqueza. Los blancos vinieron con la noticia de que la tierra donde vivian los aguarunas era tierra del Estado y no de los aguarunas... En ese tiempo los aguarunas eran analfabetos y no sabían sacar sus cuentas. Poco a poco toda la sociedad cayó en poder de los patrones por las deudas que contralan con ellos. (Wipio Deicat en Larson, Davis y Ballena Dávila, 1979 : 99-100).

Para la mayoría de los grupos nativos de la Selva, el contacto con la cultura mestiza es una rcalidad continua. Tienen que tratar con hispano-hablantes, hacer trámites para obtener los títulos de sus tierras y otros derechos para sus comunidades, y hacer otros tratos comerciales.

Por otro lado, y esto es muy legítimo, la gente en su mayoría desea que sus hijos logren la posición económica y social, y adquieran la habilidad lingüística que ofrece la sociedad mestiza. No quieren desaparecer a causa de las epidemias ni ser explotados permanentemente por las maniobras legales de otros. El ILV cree firmemente que todo grupo tiene derecho a la autodeterminación cultural, y que no se le debe negar la oportunidad de desarrollarse de la manera que le parezca ventajosa.

Es decir que los grupos étnicos de la Amazonía peruana se encuentran en una situación que cs común para las minorias lingüisticas del mundo:

Puesto que los hablantes de lenguas minoritarias, en muchos casos, tienen que volverse bilingües si desean retener su propia cultura y participar de manera significativa en la vida de la comunidad mayoritaria, es fácil comprender que la mayora de los bilingües del mundo son bilingües porque se ven obligados a serlo. Muchas de las lenguas minoritarias no ocupan la misma posición, no tienen los mismos derechos oficiales que las lenguas mayoritarias; muchas de las lenguas minoritarias son discriminadas y sus hablantes sufren opresion, tanto en las esferas políticas como económicas, y en el campo lingüístico. El bilingüismo no es para ellos una opción voluntaria sino forzada si desean sobrevivir dentro de la comunidad mayoritaria. (Skutnabb-Kangas, $1981: 745$, traducción de la autora).

Un contacto de este tipo con la cultura y la lengua hispanas conlleva un peligro adicional que ha producido daños casi irreparables en algunos grupos: el rechazo de la cultura y la lengua nativas, rechazo que se genera en el desprecio que les muestra la sociedad mestiza.

El ILV, como organización, se ha abocado a la preservación de lenguas y culturas nativas. Este compromiso se manifiesta en la prioridad que se da a la publicación de análisis lingüísticos, diccionarios bilingủes, estudios antropológicos y del folklore, y la claboración de libros de alfabetización y de lectura en los idiomas de la Selva. Sin 
embargo, el ILV considera que el ideal de la "cultura nativa intacta" no refleja la rcalidad para estos grupos. El ILV procura no impedir ni forzar cl cambio:

El ILV opina que en todo caso la ayuda prestada a un grupo étnico debe ser en respuesta a necesidades sentidas y expresadas por el grupo mismo. Así, en vez de imponer proyectos, se presta colaboración para que puedan alcanzar sus aspiraciones. (Larson, Davis y Ballena Dávila, 1979: 427).

El respeto a la autodeterminación de un grupo nativo se manifiesta, además, cuando se le ofrecen alternativas: la opción de permanecer pacíficamente en su comunidad y cultura manteniendo el intercambio necesario con la sociedad mayoritaria; o la opción que involucra ciertos aspetos de la cultura mayoritaria, cuando la comunidad considera que ello sería ventajoso. Estas alternativas se ofrecen por medio de la educación bilingüe, así como por medio de la traducción de documentos importantes y de alto valor moral como la Constitución Política del Perú, la Declaración Universal de Derechos Humanos y las Sagradas Escrituras.

\section{La educación bilingüe y su contexto social}

La perspectiva del ILV en cuanto a la educación bilingüe incorpora elementos provenientes de dos teorias diferentes o "paradigmas" de los que se habla en publicaciones actuales: el "paradigma del equilibrio" y el "paradigma del conflicto" (Paulston, 1978).

El paradigma del equilibrio sostiene que el problema inherente a la educación entre grupos minoritarios es que el ambiente educativo provee oportunidades iguales para los que no pertenecen a la lengua y cultura mayoritarias. Este paradigma hace hincapié en las relaciones entre la lengua y la adquisición de conocimientos, y por lo tanto en la necesidad de que los niffos aprendan en la lengua que dominan. La meta final del modelo del equilibrio es "la integración armónica dentro de la socicdad mayoritaria que se logra mediante la nivelación de las oportunidades" (Paulston, 1978 : 238, traducción de la autora).

Este paradigma refleja algunas de las perspectivas del ILV. Desde los primeros años del trabajo del ILV en la educación entre las minorias étnicas del Perú, se ha reconocido la desventaja del niño nativo en las aulas donde se enseña en castellano. No cabe duda que el niño nativo posee la misma capacidad de aprendizaje que el niño mestizo, pues cuando se le enseria en el idioma que comprende, aprende rápidamente y con la misma habilidad que cualquier estudiante.

La perspectiva del ILV se aparta del paradigma del equilibrio en que la mayoría de los programas de educación bilingüe que lo emplean son de tipo transicional: es decir 
que emplean "la lengua nativa del estudiante para impartirle enseñanza hasta que adquiera el dominio de [la lengua oficial]" (García y Padilla, 1985 : 291). La meta principal de la educación bilingüe transicional es la redistribución de oportunidades para aminorar las actitudes discriminatorias del pasado, pero se habla muy poco de la preservación de la lengua nativa y la cultura. Muchos de los programas de educación bilingủe que se han desarrollado en los Estados Unidos en las dos últimas décadas son un buen ejemplo de este típo de educación bilingüe transicional donde el dominio del inglés por parte de los niños de los grupos minoritarios, en los primeros años de educación, es la meta principal; no se da ninguna consideración a la preservación de su cultura y su lengua nativa.

Aquí se aparta la filosofía del ILV del paradigma del equilibrio, y encuentra puntos paralelos con el paradigma del conflicto. Este último da énfasis a la importancia de los factores sociales en la educación de las minorías. Según los que propugnan esta teoría, la desigualdad de las oportunidades educativas no se relacionan tanto con el fracaso de las escuelas como con la desigualdad social.

Desde la perspectiva del paradigma del conflicto, los factores sociales ejercen influencia sobre el éxito o el fracaso de la educación; por lo tanto el contexto del aprendizaje se convierte en un factor tan importante como la elección del idioma que se emplea (Paulston, 1978 : 244). Un niño que pertenece a un grupo minoritario que se desenvuelve dentro de un contento cultural mayoritario carece de comprensión cabal de esa cultura; cuando ello ocurre en un aula monolingüe, el estudiante de la cultura minoritaria sufre un conflicto que afecta su aprendizaje. La cultura foránea complica y dificulta el aprendizaje, aun cuando la escuela esté ubicada en la comunidad nativa. Cuando la escuela está ubicada fuera de la comunidad del estudiante, la interferencia es aún mayor. En cambio, el aula bilingüe con un profesor bilingüe nativo disminuye la interferencia y el conflicto que sufre el estudiante.

El paradigma del conflicto, y la teoría paralela de revitalización cultural y movimiento social, favorece la preservación de la lengua y aún programas de revitalización del idioma. La meta de tales programas es emplear la lengua materna para reforzar la unidad étnica del grupo. La lengua oficial viene a ser un simple instrumento que capacita al individuo para el trabajo, mientras que la lengua materna se convierte en el vehículo de enseñanza moral y de reafirmación de la solidaridad del grupo étnico (ibid.: 252).

La perspectiva del ILV se vale de algunos elementos del paradigma del conflicto/revitalización cultural. La desigualdad social, la opresión económica y aun la desintegración cultural son aspectos muy trágicos pero muy reales en la vida de muchas comunidades nativas. El ILV no considera que la educación por sí sola pueda resolver estos problemas graves que afectan al nativo, pero si que un programa educativo que hace hincapié en la preservación del idioma y el respeto a la cultura puede disminuir los efectos negativos del imperialismo cultural, el conflicto social y la explotación económica.

En estos criterios se basa también el apoyo que el ILV da a la idea de que en las escuelas bilingües los profesores sean profesores nativos. Los profesores hablan la lengua materna de alumnos y además son parte de la comunidad nativa y de la cultura. El alumno experimenta poco conflicto y la interferencia es mínima en un aula 
de este tipo; esto facilita el aprendizaje tanto en el aspecto social como lingüístico. Un profesor bora dijo al respecto:

Nosotros, como somos auténticamente vivientes de la comunidad, conocemos todo el am biente y podemos trabajar y desempeñar de lo mejor. (Gerardo del Aguila Miveco, 1989).

De lo dicho se desprende que la perspectiva del ILV presenta aspectos de más de un modelo de educación bilingủe. El modelo del equilibrio refleja el valor que el ILV da a la educación temprana en la lengua vernácula y la necesidad de buscar algún tipo de interacción armoniosa con el mundo no indígena de habla hispana; el paradigma del conflicto refleja que el ILV está al tanto de las profundas necesidades sociales y económicas que propician la desventaja educativa del niño indígena; y la teoría de la revitalización cultural refleja la importancia que se da al hecho de que el niño debe desenvolverse en un contexto que le es comprensible y con un profesor que comunica los valores culturales así como los conocimientos curriculares.

\section{El billingüismo}

Considerando lo dicho hasta aquí, se podría llegar a la conclusión de que la lengua materna es útil solamente para transmitir valores y que el castellano podria emplearse exitosamente para transmitir los conocimientos "académicos". Sin embargo, la investigación reciente del bilingüismo y la adquisición de conocimientos muestran que ésta no es una conclusión correcta; por el contrario, la investigación apoya el empleo de la lengua materna en la enseñanza de las asignaturas y para transmitir los valores culturales.

Según Baker (1988), las teorías actuales sobre el bilingüismo sostienen que los procesos cognoscitivos son los mismos para la lengua materna y para el segundo idioma. Los niños que aprenden una segunda lengua no perjudican su habilidad para retener el idioma materno; por el contrario, es posible que su habilidad cognoscitiva sea mejor que la de los niffos monolingües.

Pero Baker afirma, además, que el proceso de aprendizaje puede resultar desfavorecido si se obliga al niño a trabajar en una segunda lengua de la que tiene muy poco dominio. Si se le obliga a emplear en la escuela una lengua que apenas conoce, el conocimiento que internaliza será cuantitativa y cualitativamente más pobre. Más aún, sostiene que "los niños que poseen cierta facilidad conversacional en la segunda lengua pueden dar la impresión de que están listos para aprender por medio de la segunda lengua", pero la realidad puede ser otra; puede ser que "no entiendan significados y que no puedan realizar actividades como la síntesis, el análisis, la evaluación y la interpretación" (Baker,1988 : 179, traducción de la autora).

En algunas comunidades nativas es muy evidente que el alumno no aprende cuando el profesor es un hispano-hablante monolingule. Como los alumnos pueden 
conversar con el profesor en castellano, éste supone que puede emplear el castellano como único medio de instrucción. Luego el profesor, o la profesora, se sorprende al notar que los alumnos no aprenden. Paralelamente, en la misma comunidad, un profesor nativo bilingüe enseña otra clase en la lengua materna con resultados muy favorables. Es, pues, evidente que no es por falta de inteligencia que los niños no aprenden cuando se les enseña en castellano, sino porque su dominio del castellano es incipente.

Lo que Baker ha descubierto constituye un argumento fuerte en favor de una educación que emplea la lengua materna como medio de instrucción durante varios años y que incorpora el castellano como segunda lengua como un curso. En la mejor de las circunstancias el profesor bilingüe estaría capacitado para ayudar a los niños a desarrollar su habilidad cognoscitiva en la lengua materna y lograr un nivel de dominio del castellano que les permitiría aprender también en esa lengua.

\section{La educación bilingüe intercultural}

Las investigaciones recientes sobre la planificación de programas de educación bilingüe han dado bastante énfasis al aspecto cultural de los mismos, y para ello han empleado términos como "bicultural" e "intercultural", según el criterio de los investigadores. En su estudio sobre la educación bilingủe en la América Latina, Utta von Gleich (1987) describe la educación bilingüe/bicultural en los siguientes términos:

Presupone, además del uso de dos lenguas en el proceso educativo, la consideración sistemática, fijada en forma curricular, de las dos culturas que se hallan en contacto. (von Gleich $1987: 51$ ).

La meta de la educación bilingüe/bicultural es que el estudiante aprenda a comprender una lengua y una cultura diferentes de las propias. De esta manera, puede aceptar y sentirse cómodo en la segunda cultura al mismo tiempo que se fortalece el aprecio que tiene por su propia cultura.

La educación bilingüe/intercultural, segũn von Gleich, es un término casí equivalente al de educación bilingüe/bicultural en su aplicación a sociedades multilingủes. La posición del Perú como una nación multilingủe y multicultural sugiere que educación bilingủe/intercultural sería el término más apropiado para describir las normas y los programas que se necesitan para satisfacer las necesidades educativas del país.

La Dirección General de Educación Bilingüe del Ministerio de Educación (DIGE. BIL) describe a la educación bilingüe/intercultural de la siguiente manera: 
... aquella que además de formar sujetos bilingủes con óptima competencia comunicativa en su lengua materna y en castellano, posibilita la identificación con su cultura de origen y el conocimiento de otras culturas minoritarias y mayoritarias. ... Con esto se aspira a equiparar las posibilidades, a entender el orgullo por la cultura propia y fomentarlo, y a posibilitar que el educando se oriente entre las otras culturas con seguridad y confianza en si mismo. (Dirección General de Educación Bilingüe, $1989: 11$ ).

Como entidad que opera en estrecha relación con el Ministerio de Educación, el ILV acepta el modelo de educación bilingüe/intercultural definido por la DIGEBIL y trabaja de acuerdo con él. La definición anterior refleja además el compromiso del ILV de presentar alternativas a los miembros de las comunidades nativas que les permitan entrar en contacto positivo con la sociedad mayoritaria en la medida que consideren conveniente.

El ILV, sin embargo, a la vez que apoya las alternativas educativas que permiten un menor conocimiento de la cultura mayoritaria, reconoce que la mayoría de las culturas amazónicas corren el inminente peligro de ser asimiladas por la cultura que las rodea y por ende, de desaparecer. Skutnabb-Kangas, al hablar de los idiomas de las minorias, dice:

La posición de las lenguas minoritarias está en peligro en todo el mundo, aun en las naciones donde muchas de ellas se reconocen oficial o semioficialmente... Esos grupos idiomáticos se encuentran en una posición muy dif́́cil cuyo único apoyo proviene de una lengua no estandarizada... o de una lengua vernácula de otro país. Sin embargo, los grupos idiomáticos que no poseen tradición escrita, que se hablan en un solo pals y que no cuentan con el apoyo de hablantes de otros paises, son los más amenazados. Este es el caso de muchas lenguas indigenas americanas que se hablan en los Estados Unidos, el Canadá, la América Latina, y también algunas lenguas del Africa y el Asia. La actitud hacia estas lenguas y grupos idtomáticos puede ir desde tratar de proveerles un alfabeto, grabarlas, describirlas y elaborar materiales elementales de lectura... por un lado, hasta intentos de erradicarlas, por el otro. (1981:71, 74, traducción de la autora).

La posición precaria e inestable de las minorías étnicas en la Amazonía peruana es la razón que justifica un apoyo decidido a la supervivencia de su lengua y su cultura ante la amenaza de una sociedad mayoritaria que se acerca a ellas cada vez más.

Por ello, el ILV da más atención al aspecto vernáculo de la educación bilingủe que al aspecto de la familiarización con la segunda cultura. Las lenguas vernáculas y los grupos étnicos son los campos en que se han especializado sus trabajadores de campo, y es precisamente en estos campos que el ILV está capacitado para complementar el programa de educación del Estado. Como parte del apoyo que se ofrece al Ministerio de Educación, el ILV elabora y apoya en la distribución de libros de lectura y ayuda a los maestros bilingües a aplicar las técnicas pedagógicas apropiadas en las comunidades nativas. El ILV apoya los diferentes aspectos del programa de educación bilingüe del Ministerio de Educación pero concentra su atención en la preparación de materiales en el vernáculo y en la capacitación de los maestros bilingües. 


\section{Una apreciación crítica}

La evaluación meșrada revela que la educación bilingüe, sin importar el modelo que siga, no es por sí sola la solución para satisfacer todas las necesidades sociales, económicas, políticas y educativas de los grupos indigenas del Perú. La educación bilingüe por sí sola no puede propiciar estabilidad cultural, ni la interacción saludable. entre las culturas nativas y la cultura mayoritaria. La educación bilingủe es solamente un instrumento. El ILV, sin embargo, está convencido de que un programa educativo orientado a la supervivencia de las lenguas vernáculas y de carácter bilingủe/intercultural como el descrito en el presente trabajo ha probado ser un instrumento muy útil para promover la revaloración de la cultura y para legar a las generaciones futuras un sentimiento de autoestimación.

Si comparamos este modelo de educación bilingủe con las alternativas monolingủes -ya sea en la lengua nativa o en la lengua de la mayoria- podemos notar que aquél ofrece muchas ventajas para el nativo. Desde el punto de vista cultural, las ventajas de la educación bilingüe sobre la monolingüe en castellano son que fomenta la unidad del grupo étnico (pues disminuye las posibilidades de una dominación cultural y linguística por parte de foráneos); y que ayuda a mejorar el prestigio de las lenguas y culturas indígenas, y por lo tanto a mejorar el sentido de dignidad del individuo. Sánchez Garrafa expresa esto en los siguientes términos:

La utilización de la lengua vernácula en el proceso educativo constíluye una medida concreta de revaloración y por tanto un estímulo psicológico para la pobladón vernáculo hablante en el mejoramiento de un auto-concepto o autovaloración. (1983:41).

Las comunidades nativas y los profesores bilingües están muy conscientes de estas ventajas culturales de la educación billingủe. Para un profesor bilingüe shipibo "ha sido un despertar educativo de los niños, ponque se han orientado a la valoración del idioma y (las) costumbres" (expresión anónima recogida en una encuesta en 1989). Un profesor asháninca afirma también que por medio de la educación bilingüe "se valoró las culturas y se rescató lo perdido; y hay mucha relación entre los padres, alumnos y el profesor" (ibid). Los trabajadores de campo del ILV han notado que los grupos nativos que están reconociendo el valor de su herencia cultural son los que solicitan la creación de más escuelas bilingües como parte de su despertar cultural.

Las ventajas pedagógicas de la educación bilingủe sobre la educación monolingủe en castellano están relacionadas con la adaptación psicológica del alumno y con la facilidad para aprender las asignaturas que se le ensenan en la lengua materna. Engle (1975) dice lo siguiente en cuanto a las ventajas de emplear el vernáculo en la instrucción inicial:

Puesto que el niño puede comprender lo que se le dice, tiene muchas probabilidades de un éxito que lo ayudará a desarrollar un sentimiento de competencia en el aula. $(1975: 4$, traducción de la autora).

A esto podemos añadir que el nifno que aprende a aprender -es decir, que adquiere los procesos cognoscitivos que se necesitan para analizar, evaluar e interpretar- en su 
lengua materna construye un fundamento sobre lo que puede basar el resto de su educación (Baker, 1988: 179).

Los aspectos biculturales de la educación bilingüe le dan ventaja sobre la educación monolingủe en el vernáculo. Los padres de los niños nativos, en su mayoría, desean que sus hljos aprendan el castellano para poder cómunicarse con los miembros de la sociedad mayorltaria, Y ésta podria ser la razón más importante por la que desean la educación de sus hijos. La situación que Wipio Diecat describe y que determinó la decisión de los aguaruna de procurar la educación es aún un sentimiento fuerte entre los grupos nativos:

Los patrones tenían sus libros en donde anotaban todas las cosas que entregaban a los aguarunas, pero cuando un aguaruna traía un producto [los patrones] no hacian el descuento.... En csta forma los aguarunas iban pensando algo sobre el estudio. [Querfan] aprender a escribir, leer y hablar el castellano para que pudieran reclamar sus derechos y pudieran vender sus productos sin que nadie les engañara. (Wipio Deicat en Larzon, Davis y Ballena Dávila, $1979: 101$ ).

Por esta razón, un programa de educación monolingüe en el vernáculo no sería popular y quizá hasta sería rechazado por los vernáculo-hablantes de la Selva.

La educación bicultural o intercultural, además, amplía la cosmovisión del alumno pues le permite comparar su propia cultura con la de otros y escoger su propio modo de vida. Según la DIGEBIL, una de las funciones de la educación bilingüe/intercultural es que "permite al educando discernir respecto a los elementos de otras culturas que podrán constituir un valioso aporte para el mejoramiento cualitativo de su nivel de vida y de su comunidad, erriqueciendo así su propia cultura". (DIGEBIL, 1989 : 11).

Al respecto, es necesario tomar en cuenta que la educación bilinglie no se implementa con la misma facilidad con que se desarrolla un programa monolingủe, en especial la educación monolingủe en castellano. Los centros educativos bilingües requieren de personal docente especial y textos escolares especiales y su implementación es más costosa.

El profesor bilingüe nativo es el elemento clave para el éxito de la educactón bilingủe, y la persona que ocupa dicha posición debe tener un trasfondo especial, asimismo cualidades y capacitación que van más allá de las que se exigen normalmente de un profesor. Debe ser un individuo aceptado por su comunidad, debe ser nativo hablante del idioma de la comunidad y debe saber suficiente castellano para desempentar su trabajo. Debe, además, recibir capacitación pedagógica para utilizar correctamente los textos bilinglies disponibles. Debe estar comprometido con el progreso de su grupo, aun en lo que respecta la preservación de la lengua y la cultura. Por ejemplo, una mujer piro dijo que "un buen maestro bilingüe enseña bien a los alumnos y se precocupa por el bienestar de su comuridad" (encuesta realizada en 1989).

Muchos de los maestros bilingües que actualmente trabajan en sus comunidades ciertamente se han comprometido de esa forma con su trabajo. Un profesor shipibo expreso el deseo de que "mis amigos y mis alumnos no olviden ni descuiden su cultura". Otro explica que "las dos lenguas son importantes; debemos sentirnos orgullosos". Un profesor cashinahua considera que la educación bilingủe es importante "para com-

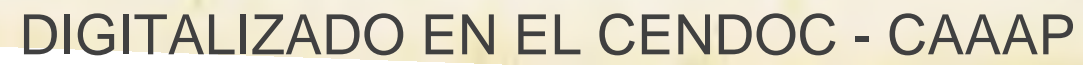


prendernos los unos a los otros y también a los que hablan castellano" (encuesta realizada for Wipio Deicat en 1989 ).

Otro requisito especial de este tipo de educación bilingüe es que el alumno pase más horas en el aula. El curso de lenguaje en el currículo bilingüe se divide en dos asignaturas, "lengua materna" y "castellano como segunda lengua", cada una de las cuales toma una hora diaria. De manera que el horario de la escuela bilingüe tiene una hora más para que el alumno reciba suficiente instrucción en la lengua materna y en la segunda lengua.

Finalmente, el programa de educación bilingüe es más costoso que un programa monolingủe en castellano. Cada uno de los grupos indigenas necesita suficientes textos para el aprendizaje inicial de la lectura, para la práctica de la lectura, y otros textos como de ciencias y salud. La meta de los especialistas en educación bilingüe y de los linguistas del ILV es elaborar por lo menos seis libros de lectura en el vernáculo para cada uno de los grupos; muchos de ellos cuentan en la actualidad con más de doce títulos, y algunos tienen más de veinte. La producción, reimpresión y distribución de los textos bulingües aumenta considerablemente los costos en comparación con un sistema que empler los textos ya existentes en castellano. La capacitación de los profesores nativos en cursus de pedagogia bilingüe representa gastos adicionales.

Si se lo compara con un modelo de educación monolingìe en el vernáculo, el programa de educación bilingủe presenta las mismas dificultades de entrenamiento, tiempo y de los recursos económicos que se necesitan; pero se debe notar que un programa monolingle en el vernáculo además tendría que considerar la elaboración de materiales educativos en el vernáculo para los grados avanzados.

\section{Conclusión}

Se ha dicho, pues, que la perspectiva del ILV en lo que se refiere a la educación bilingüe se basa en el nespeto por las lenguas y las culturas nativas, así como en el reconocimiento de que existe una "presión externa" que las sociedades indígenas deben poder afrontar. El ILV se ha comprometido a apoyar el programa de educación bilingule oficial y su contribución se concentra en el aspecto vernáculo del mismo.

El sistema de educación bilingüe ha avanzado mucho desde su creación en 1952. Esto se puede notar fácilmente entre los grupos que han participado en el programa por más tiempo; grupos en los que los profesores bilingües actuales pertenecen a la segunda o tercera generación enseflan mejor que sus antecesores. La supervisión y capacitación de los profesores bilinglies, que aún se ven limitadas por el aislamiento de muchas comunidades, están poco a poco pasando a ser una responsabilidad de especialistas nativos que poseen la capacitación y el trasfondo cultural para ayudar a los profesores a de- 
sempeñar mejor su papel. La graduación de la primera promoción del Instituto Superior Pedagógico Bilingüe en 1989 representa otro aspecto del progreso -el de la educación superior para los profesores nativos.

No ha disminuido la necesidad de una educación en el vernáculo. Un porcentaje bastante alto de la población indígena de la selva es aún monolingüe o es bilingüe incipiente. La llegada de colonos y otros foráneos sigue siendo una amenaza para la estabilidad cultural, y por ello la educación, que se preocupa por la preservación del idioma y la cultura y que presenta alternativas positivas a los grupos nativos, sigue siendo una necesidad imperiosa para la supervivencia de dichas culturas.

Por esta razón, el ILV se une con el Ministerio de Educación en los esfuerzos para alcanzar la meta ideal de que la Amazonía peruana cuente con un programa bilingüe auto-suficiente. Deben ser los mismos nativos los que se propongan mantener un sistema educativo vital y próspero. Ellos son los que conocen mejor las necesidades de la comunidad y son los que a la larga resultarán favorecidos con los resultados de un programa de educación bilingüe.

Para lograr esta meta es preciso capacitar a los nativos que desempeñan los cargos de profesores, capacitadores y administradores dentro de la infraestructrura educativa. El proceso ya se ha iniciado y avanza a medida que el Ministerio de Educación da prioridad a los cursos de capacitación y nombra a profesores y especialistas nativos para ocupar importantes cargos dentro del sistema de educación bilingüe.

El programa de educación bilingüe de la Amazonía dista mucho de ser perfecto; los obstáculos que hay que vencer son muchos y se reconoce que el éxito logrado por las escuelas bilingües de las comunidades es modesto. Sin embargo, se ha avanzado $y$ ahora, más que nunca, se vislumbra la posibilidad de que el programa se convierta en un programa autosuficiente. 


\section{Bibliografia}

BAKER, Colin, 1988. Key issues in bilingualism and bilingual education. (Multilingual matters 35.) Avon: Multilingual Matters Ltd.

DECLARACION UNIVERSAL DE LOS DERECHOS HUMANOS. 1948. New York: Naciones Unidas.

DEL AGUILA MIVECO, Gerardo. Entrevista personal, marzo de 1989.

DIRECCION GENERAL DE EDUCACION BILINGÜE.' 1989. Política de educación bilingüe intercultural. Lima: Ministerio de Educación.

ENGLE, Patricia Lee. 1975. The use of vernacular languages in education: Language medium in early school years. for minority language groups. Arlington: Center for Applied Linguistics.

GARCIA, Eugene E. y Raymónd V. Padilla, eds. 1985. Advances in bilingual education research. Tucson: University of Arizona Press.

LARSON, Mildred L. Patricia M. Davis y Marlene Ballena Dávila, eds. 1979. Educación bilingüe: Una experiencia en la Amazonía Peruana. Lima: Ignacio Prado Pastor.

MINISTERIO DE EDUCACION. 1970. Reforma de la Educación Peruana: informe general. Lima: Ministerio de Educación.

PAULSTON, Christina Bratt. 1978. "Theoretical perspectives on bilingual education programs". International dimensions of bilingual education, James' A. Atlatis ed., pp. 230-67. Washington, D.C.: Georgetown University Press.

SANCHEZ GARRAFA, Rodolfo. 1983. Perspectivas para una educación bilingüe para adultos. Lima:INIDE.

SKUTTNAB-KANGAS, Tove. 1981. Bilingualism or not: the education of minorities. (Multilingual matters 7.) Avon: Multilingual Matters Ltd.

VON GLEICH, Utta. 1987. Educación primaria bilingüe y bicultural en América Latina. (Informe de Educación NN 34). Eschborn, Germany: Deutsche Gesellschaft fur Technische Zusammenarbeit.

WIPIO DEICAT, Gerardo. Encuesta anónima e inédita realizada entre los profesones bilingües en febrero de 1989. Instituto Superior Pedagógico Bilingüe, Yarinacocha, Perú.

WIPIO DEICAT, Gerardo. Encuesta inédita realizada en las comunidades nativas en agosto de 1989. 